\title{
New psychological treatments in schizophrenia
}

\section{Gillian Haddock \& Shôn Lewis}

The first line of treatment for patients with psychotic disorders such as schizophrenia is neuroleptic medication. Neuroleptics have provided substantial benefits to patients with this type of severe mental illness since their discovery as a treatment for psychosis in the 1950s. Despite this, there are still a large number of patients who do not respond fully to neuroleptic medication or who are not able to tolerate it. For example, although as many as $70 \%$ of patients are substantially improved following drug treatment, a considerable proportion continue to experience persistent, distressing and recurrent symptoms. In a survey of patients in a London psychiatric hospital, Curson et al (1988) found that just under half of the patients continued to experience hallucinations and delusions despite the prescription of medication. In addition, many patients experience intolerable side-effects or do not wish to comply with neuroleptic medication, yet look for some effective alternative. Depression, anxiety and a high rate of suicide are additional problems faced by patients with schizophrenia.

As a result of this, psychological treatments for schizophrenia and other psychotic disorders have been developing over the last decade, particularly in the area of cognitive-behavioural interventions. Recent research has focused on the following areas: (i) family interventions aimed at improving patient and carer functioning and reducing relapse; (ii) individual cognitive-behavioural interventions aimed at reducing the severity of drug-resistant psychotic symptoms; (iii) attempting to teach patients and their carers to monitor prodromal signs to facilitate early intervention, and (iv) strategies aimed at increasing compliance to medication. This paper will briefly review each of these areas and the implications for training and service provision will be discussed. It will not attempt to cover more traditional psychological approaches such as social skills training or token economy approaches for negative symptoms (see Kavanagh, 1992a for a review of these areas).

\section{Family interventions}

Following the observations of George Brown in the 1950s, that some schizophrenic patients discharged to certain environments were more likely to relapse than others, much research has focused on the family environment as a predictor of relapse. Brown \& Rutter (1966) introduced the concept of 'expressed emotion' (EE) to describe those environments which most contributed to relapse. A high EE environment (as measured by the Camberwell Family Interview), consists of relatives who demonstrate high levels of criticism, hostility and emotional overinvolvement towards their schizophrenic relative. Patients living in high EE environments have been shown in a number of studies to have a significantly higher relapse rate than those patients living in low EE environments (see Kavanagh, 1992b for a review).

In addition, Vaughn \& Leff (1976) observed that the amount of contact with high EE relatives and whether the patient was taking medication were both factors which interacted with high EE and predicted relapse. Patients who were not taking medication and who spent more than 35 hours per week with their high EE relative had a high relapse rate over 9 months (92\%). Those who had similar contact but who were taking medication showed

Gillian Haddock is an Honorary Consultant Clinical Psychologist for South Manchester University Hospitals NHS Trust and tutor in Clinical Psychology at the School of Psychiatry and Behavioural Sciences, University of Manchester, Withington Hospital, West Didsbury, Manchester M20 8LR. Shôn Lewis is Professor of Adult Psychiatry at the University of Manchester and South Manchester University Hospitals NHS Trust. 
an intermediate relapse rate over a similar period (53\%), as did those with less contact but who were not taking medication (42\%). Those with less contact and who were taking medication showed a low relapse rate (15\%), as did patients who were living in low EE environments. In low EE environments, the relapse rate was relatively unrelated to whether the patient was taking medication or not (12\% and $15 \%$ respectively).

Following these observations, many researchers have examined whether family interventions could reduce the EE status of relatives and whether this has a consequential effect on relapse rates. A number of studies (e.g. Tarrier et al, 1988) have shown that family interventions for patients with a diagnosis of schizophrenia and their families, in addition to treatment as usual, could reduce the relapse rate of those patients, increase their social functioning and reduce subjective burden in their families. The benefits of these approaches have also been shown to be apparent up to 8 years after the intervention (Tarrier et al, 1994). In a recent metaanalysis of the best six published studies (involving a total of 350 patients, 181 controls and 169 experimental subjects), De Jesus Mari \& Streiner (1994) showed that 9 months following the implementation of a family intervention there was a marginally significant superior effect for family interventions over controls on patient relapse rates. Patients receiving family intervention also showed increased drug compliance and lower overall hospitalisation than controls. In addition, families of patients receiving a family intervention were more likely to become low EE over 9 months of treatment compared with the families of control patients.

The family interventions differed slightly in each of the published studies but all have a number of common components. The interventions have usually been behavioural or cognitive-behavioural in orientation (a psychoanalytically oriented approach carried out by Kottgen et al (1984) in Hamburg failed to demonstrate that the family intervention had any benefit over routine treatment in terms of subsequent relapse rates). Most interventions have consisted of education regarding the nature of the illness, its treatment, management and prognosis, together with strategies to reduce stress in the family, increase independence and to solve problems generated during day to day life. Most interventions have taken place over 9 months and some have provided booster sessions for families and patients which increased the benefits over longer periods (Falloon et al, 1985).

Despite the research findings, the integration of family interventions into routine services for patients has not occurred. Why is this? It may be due to a lack of training in family interventions or a view that these types of intensive treatments are more expensive than conventional ones. Although it is true that family interventions require trained professionals who will provide intensive input, this is generally over a relatively short period of time in the career of a schizophrenic patient and their family. Therefore, the economic savings made by reducing future relapse rates can easily justify any initial costs. With regard to training the appropriate staff to deliver these interventions, recent research has demonstrated that community psychiatric nurses are in a good position to deliver the interventions and can be trained to do so, resulting in improved patient and carer outcomes. In a recent study by Brooker et al (1992), two groups of CPNs were taught assessment techniques and trained to deliver family interventions. Improvements were found in positive and negative psychotic symptoms as well as in social functioning of patients. Relatives' satisfaction with services increased and reports of their own minor psychiatric morbidity improved.

\section{Individual psychological management of psychotic symptoms}

Many individuals with a diagnosis of schizophrenia continue to have residual positive and negative symptoms which have not responded to medication. These persistent symptoms can contribute to continued distress, reduced social functioning and may increase the risk of self-harm (Falloon \& Talbot, 1981). Traditionally the medical model in psychiatry has stressed the Jasperian notion of the 'non-understandability' of symptoms in schizophrenia (Jaspers, 1963). This has carried the implication that psychological treatments will, by definition, not work. For some psychological treatments this may well be the case: for example, psychoanalytically oriented psychotherapy has not been shown to be effective with schizophrenic patients (Meuser \& Berenbaum, 1990).

Nonetheless, recent studies examining the efficacy of cognitive-behavioural approaches in the management of drug-resistant symptoms have shown that these can be successful at reducing the occurrence of symptoms and the distress associated with them. The majority of early studies have been in the form of individual case studies or case series (reviewed by Haddock et al, 1994). The approaches 
described have varied from operant procedures designed to provide reinforcement when the patient is engaged in non-psychotic behaviour or speech (Wincze et al, 1972); distraction techniques designed to reduce auditory hallucinations (Margo et al, 1981); belief modification approaches designed to alter the characteristics of delusions (Chadwick \& Lowe, 1990) and systematic desensitisation to the factors which contribute to the occurrence of symptoms (Slade, 1972).

Until recently, many authors have focused on single behavioural or cognitive techniques in order to reduce the severity of the psychotic symptoms. Researchers have now begun to see the need for controlled studies with larger numbers of subjects (Tarrier et al, 1993) in order to demonstrate the efficacy of psychological approaches. Such studies are starting to demonstrate that cognitive-behavioural approaches are significantly better at reducing the severity of persistent psychotic symptoms than conventional treatment alone or comparison control groups offering alternative individual approaches (Garety et al, 1994).

\section{Prodromal signs monitoring and early intervention}

The natural history of schizophrenia is typically one of repeated relapse of acute psychosis. Birchwood et al (1989) have demonstrated that it is possible to train patients and their carers to identify early signs which may herald a relapse in order to intervene to reduce the severity of the relapse. They have observed that many patients have individually characteristic changes in affect and cognition which precede a full blown relapse, although there is much variation in the characteristics of these prodromes between patients. The term 'relapse signature' describes this and demonstrates that through regular and frequent monitoring of symptoms and behaviours it is possible to identify the early signs which are likely to herald a relapse. Early intervention can then be directed to reducing the likelihood of relapse using increases in neuroleptic medication, support and cognitivebehavioural techniques.

There appears to be a small amount of evidence that this type of early intervention can be effective providing the monitoring process is frequent enough to identify the time window between 'wellbeing' and a subsequent relapse. For some patients this can be over a matter of days, whereas for others it can be over a number of months. Birchwood suggests that monitoring should occur at least fortnightly for the majority of patients, if the time window is unknown, as this has been shown to identify approximately $75 \%$ of patients who go on to a full blown relapse.

\section{Increasing patient adherence to medication}

Non-compliance with neuroleptic medication is an important contributory factor in prolonged psychosis and the occurrence of relapse. In the study by Vaughn \& Leff (1976) reported above, patients who refused medication for one month out of a nine month follow-up period had approximately three times the relapse rate compared with those who remained fully compliant. Although it could be speculated that non-compliance in patients who are severely mentally ill is related to the nature of their illness, it has been shown that non-compliance is no more prevalent than in other illnesses which require long-term medication treatment and can sometimes be viewed as a marker of health rather than illness (Ley, 1992). Factors which have been identified as contributing to non-compliance include complex drug regimes, demographic factors (Sellwood \& Tarrier, 1994), level of symptomatology (Bartko et al, 1988), insight (Bartko et al, 1987) and attitudinal factors (Ludwig et al, 1990). Poor compliance can be secondary to side-effects such as sedation, akathisia or insight gain. Seltzer et al (1980), have also found that fear of side-effects was associated with noncompliance, but that if patients were provided with education about the nature of side-effects, this reduced their fear without affecting compliance. Non-compliance is possible both with oral and depot drugs, but covert non-compliance is only possible with oral drugs.

Recent approaches which have been used to increase compliance have focused on providing education regarding the nature of side-effects and medication (Eckman \& Liberman, 1990), establishing a collaborative approach (Corrigan et al, 1990) and approaches which have attempted to teach patients the skills needed for compliance, for example, memory aids (Eckman et al, 1990). More recently, Sellwood et al (1994) have been investigating the effectiveness of motivational interviewing as an approach for use with noncompliant patients. Motivational interviewing is a collaborative approach which involves structured assessment and education about the nature of diagnosis, medication and its potential side-effects and an emphasis on helping the patient to review 
what they have to gain or lose by not accepting treatment and remaining ill. The therapist aims to help the patient to generate a range of possible options, of which taking medication or other treatment may be one, and examining the possible consequences of each. Although the efficacy of this with non-compliant schizophrenic patients has yet to be demonstrated, it has been shown to be effective with other groups of psychiatric patients, for example, problem drinkers (Miller \& Rollnick, 1991).

\section{Cognitive remediation of psychological deficits}

Patients with schizophrenia show a range of information processing deficits. These are thought to reflect underlying cognitive deficits implicated in schizophrenia, although the large number of information processing deficits which have been identified have not led to a clear understanding of the core deficits which are shown by all patients (Hemsley, 1993). This area is further complicated by the difficulty in distinguishing whether a particular deficit is a feature of the illness itself or whether it is a secondary consequence to other factors, such as coping with distressing symptoms. Despite this, a number of authors have attempted to identify the frequent psychological deficits found in patients with schizophrenia and develop training packages specifically to remedy the deficits and ultimately lessen their impact on behaviour and symptoms. Early advocates of this approach were Meichenbaum \& Cameron (1973) who developed an intensive programme which assumed that the attentional deficits of patients with schizophrenia arise from a failure in verbal selfregulation. They attempted to train patients to overcome this difficulty by using self-instructional training; where patients are helped to complete puzzles using self-directed verbal instructions. The authors reported that improvements were found over a range of intellectual tasks and resulted in reductions in disordered speech in the patients. Despite these findings, there have been problems in completely replicating the findings, particularly in relation to the generalisability of improvements (Bentall et al, 1987). More recently, other authors have also demonstrated improved performance of patients trained on specific cognitive tasks (e.g. Bellack et al, 1990). Despite this, these studies have shown little or no evidence that these changes generalise to any other area of functioning.
Using a slightly different therapeutic approach, Brenner et al $(1990,1992)$ developed Integrated Psychological Therapy (IPT) which combines cognitive therapy with forms of interpersonal skills training in a modular group training programme comprising five subprogrammes. The subprogrammes are arranged so that the first interventions target basic cognitive skills while later ones target complex interpersonal problems. The IPT intervention has been evaluated and compared with a placebo activity control and routine treatment and results indicated that the IPT group scored significantly higher on tests of attention than either of the two control groups and overall psychopathology decreased significantly for the IPT groups but not for the control groups. These results were maintained at 18 month follow-up, which also revealed a lower hospitalisation rate for the IPT group.

Despite these recent developments in combining cognitive therapies with social performance training and the observation that patients can be trained to improve on specific tasks, there is still a long way to go before it becomes a viable approach to treatment. First, there is no consensus among researchers as to the nature of a deficit which is common to all patients who have a diagnosis of schizophrenia and second, as a result of this there is no consensus as to which of the range of deficits demonstrated by patients should be targeted for treatment.

\section{Comprehensive treatment programmes}

In the light of the developments in the individual interventions with psychotic patients, a number of authors have combined techniques which have been shown to be individually effective to produce a comprehensive management package (Garety et al, 1994; Kingdon \& Turkington, 1994). These cognitive-behavioural interventions attempt to address the multiple needs of psychotic patients, such as depression; anxiety; lack of knowledge of their illness, medication and side-effects; reduced social functioning; negative symptoms, and strategies aimed at reducing the severity of hallucinations and delusions. In a small controlled trial employing this type of comprehensive approach, Garety et al (1994) found superior outcomes for patients receiving an average of 16 sessions of individual therapy over 6 months on delusional conviction, general symptomatology and depression. 


\section{Interventions with acute patients}

Most of the interventions described above have been delivered to patients experiencing chronic psychotic symptoms, rather than patients who are experiencing a recent onset acute episode. It could be hypothesised that if patients can be targeted in the early stages of their illness, long-term gains may be even greater. Indeed, there is some support for this. A recent study by Drury et al (1994) has shown that a comprehensive psychological treatment approach can also be appropriate for acute inpatients with a short illness history as well as patients with more chronic problems. An intensive ward-based individual cognitive-behavioural intervention together with family sessions and an eight week education programme was compared with an equal contact recreational activity control group and a second control group receiving routine treatment only. (Results indicated that the time to operationally defined recovery was reduced by 25 $50 \%$ in the experimental group as compared to the control groups.) Mean time to discharge from hospital was also approximately halved in the experimental group. The authors are currently engaged in a study to replicate these findings and to demonstrate the effectiveness of CBT solely with acute in-patients with less than a two year history of schizophrenia (Lewis et al, 1995).

\section{Implications for training}

There appears to be little doubt that psychological interventions for patients with severe mental health problems can have a large impact, not only on relapse rates, but on the severity of psychotic symptoms, length of hospital stay, affective symptoms and social functioning of carer and patient. Despite this, their dissemination into routine services is sadly lacking. Recent work being carried out at the University of Manchester and the Institute of Psychiatry, London (funded by the Sir Jules Thorn trust), attempts to provide mental health professionals with the additional skills necessary to carry out these types of psychosocial interventions. A three module diploma programme (the Thorn psychosocial intervention training) has been developed and, so far, CPNs have been taught to deliver problem oriented case management, family management and individual psychological management to individuals and their families.

Preliminary findings from students in Manchester have proved encouraging (Lancashire et al, 1996). Nurses were trained in the reliable assess- ment of mental state using an extended version of the KGV Symptom Scale (Krawiecka et al, 1977) and in the assessment of social functioning using the Social Functioning Scale (Birchwood et al, 1990); these were administered to all patients prior to Thorn psychosocial intervention and 12 months later. Pilot data from 27 patients show a significant reduction in positive, negative and affective symptoms, together with a significant improvement in social functioning (Lancashire et al, 1996). These results compare favourably with those from initial pilot studies reported by Brooker et al (1994), which assessed patient outcome in a similar way. The control sample of patients in Brooker et al's study showed no significant changes in symptoms or social functioning following 12 months of standard psychiatric nursing care.

Box 1. Key points

Although drug treatments remain the mainstay of acute treatment, many patients continue to experience psychotic symptoms.

Family interventions can significantly reduce patient relapse rates.

Persistent positive symptoms are often amenable to cognitive-behavioural interventions.

Detection of prodromal signs is possible in individual patients and may be useful to aid early intervention prior to acute relapse.

Poor compliance is multi-factorial but can often be improved with education and psychological techniques.

Neuropsychological deficits in chronic schizophrenia are identifiable and may be remediable by intensive cognitive approaches.

Despite good evidence for their efficacy, many psychological interventions have yet to be implemented into routine services.

A variety of mental health professionals are able to provide the approaches as long as they have appropriate training and supervision.

Despite research findings supporting the efficacy of psychological approaches they have not become routine in clinical practice. 


\section{Implications for clinical practice}

The main types of psychological interventions shown to be effective for patients with schizophrenia involve individual work to reduce the impact of symptoms, increase compliance with medication and improve patients' ability to detect and alert services to the start of an acute relapse, and family work aimed towards reducing the risk of relapse and increase patients' and relatives' social functioning (Box 1). Even though these are specialist types of interventions which usually require the services of a clinical psychologist it is possible to train other mental health professionals, such as CPNs, to deliver the interventions, providing they receive appropriate guidance and supervision. As a result of this, as well as the current development of new antipsychotic drugs, there can now be fresh optimism for the future of services for individuals with schizophrenia. Policy makers and purchasers of mental health care need to be assertively educated about the unmet needs arising out of these new technologies. Psychological treatments can offer an adjunct or even an alternative to conventional drug treatments. It is likely that any progress made could be considerably enhanced by the increasing interest in the viability of these approaches and the amount to which this interest is carried from researchers and clinicians to fund-holders and policy makers, who will ultimately be responsible for determining the future of services to individuals experiencing psychotic symptoms.

\section{References}

Bartko, G., Maylath, E. \& Herczeg, I. (1987) Comparative study of schizophrenic patients relapsed on and off medication. Psychiatry Research, 22, 221-227.

-, Herczeg, I. \& Zador, G. (1988) Clinical symptomatology and drug compliance in schizophrenic patients. Acta Psychiatrica Scandinavica, 77, 74-76.

Bellack, A. S., Meuser, K., Morrison, R. L., et al (1990) Remediation of cognitive deficits in schizophrenia. American Journal of Psychiatry, 147, 1650-1655.

Bentall, R. P., Higson, P. J. \& Lowe, C. F. (1987) Teaching selfinstructions to chronic schizophrenic patients: efficacy and generalisation. Behavioural Psychotherapy, 15, 58-76.

Birchwood, M., Smith, J., Macmillan, F., et al (1989) Predicting relapse in schizophrenia: the development and implementation of an early signs monitoring system using patients and families as observers. Psychological Medicine, 19, 649-656.

-_, _- Cochrane, R., et al (1990) The development and validation of a new scale of social adjustment for use in family intervention programmes with schizophrenic patients. British Journal of Psychiatry, 157, 853-859.

Brenner, H. D., Kraemer, S., Hermanutz, M., et al (1990)
Cognitive treatments in schizophrenia. In Schizophrenia: Concepts, Vulnerability and Intervention (eds E. R. Straube \& K. Hahlweg), Berlin: Springer-Verlag.

- Hodel, B., Roder, V., et al (1992) Treatment of cognitive dysfunctions and behavioural deficits in schizophrenia: Integrated psychological therapy. Schizophrenia Bulletin, 18, 21-26.

Brooker, C., Tarrier, N., Barrowclough, C., et al (1992) Training community psychiatric nurses for psychosocial intervention. Report of a pilot study. British Journal of Psychiatry, 160, 834844.

- Falloon, I. R. H., Butterworth, A., et al (1994) The outcome of training community psychiatric nurses to deliver psychosocial intervention. British Journal of Psychiatry, 165, 222-230.

Brown, G. W. \& Rutter, M. (1966) The measurement of family activities and relationships: a methodological study. Human Relations, 19, 241-263.

Chadwick, P. \& Lowe, C. F. (1990) The measurement and modification of delusional beliefs. Journal of Consulting and Clinical Psychology, 58, 225-232.

Corrigan, P. W., Liberman, R. P. \& Engel, J. D. (1990) From noncompliance to collaboration in the treatment of schizophrenia. Hospital and Community Psychiatry, 41, 12031211.

Curson, D. A., Patel, M., Liddle, P. F., et al (1988) Psychiatric morbidity of a long-stay hospital population with chronic schizophrenia and implications for future community care. British Medical Journal, 297, 819-822.

De Jesus Mari, J. \& Streiner, D. L. (1994) An overview of family interventions and relapse in schizophrenia: meta-analysis of research findings. Psychological Medicine, 24, 565-578.

Drury, V., Birchwood, M., Macmillan, F., et al (1994) Promotion of recovery from acute episodes of psychosis. Paper presented at International Symposium of Schizophrenia, Oslo, Norway.

Eckman, T. A. \& Liberman, R. P. (1990) A large scale field test of a medication management program for people with schizophrenia. Psychosocial Rehabilitation Journal, 13, 31-35.

_-, _-, Phipps, C. C., et al (1990) Teaching medication management skills to schizophrenic patients. Journal of Clinical Psychopharmacology, 10, 33-38.

Falloon, I. R. H. \& Talbot, R. E. (1981) Persistent auditory hallucinations: coping mechanisms and implications for management. Psychological Medicine, 11, 329-339.

-, Boyd, J. L. \& McGill, C. W. (1985) Family management in the prevention of morbidity in schizophrenia: Clinical outcome of a two year longitudinal follow-up. Archives of General Psychiatry, 42, 887-896.

Garety, P. A., Kuipers, L., Fowler, D., et al (1994) Cognitivebehavioural therapy for drug-resistant psychosis. British Journal of Medical Psychology, 67, 259-271.

Haddock, G., Sellwood, W., Yusupoff, L., et al (1994) Developments in cognitive-behaviour therapy for persistent psychotic symptoms, Behaviour Change, 11, 1-16.

Hemsley, D. R. (1993) A simple (or simplistic?) cognitive model for schizophrenia. Behaviour, Research and Therapy, 31, 633645.

Jaspers, K. (1963) General Psychopathology (translated by J. Hoenig \& M. W. Hamilton). Manchester: Manchester University Press.

Kavanagh, D. (ed) (1992a) Schizophrenia: An Overview and Practical Handbook. London: Chapman \& Hall.

- (1992b) Recent developments in expressed emotion and schizophrenia. British Journal of Psychiatry, 160, 601-620.

Kingdon, D. G. \& Turkington, D. (1994) Cognitive-behavioural Therapy of Schizophrenia. Lawrence Erlbaum Associates.

Kottgen, C., Soinnichesen, I., Mollenhauer, K., et al (1984) Results of the Camberwell Family Interview study, I-III. International Journal of Family Psychiatry, 5, 61-94.

Krawiecka, M., Goldberg, D. \& Vaughan, M. (1977) A standardised psychiatric assessment scale for chronic psychiatric patients. Acta Psychiatrica Scandinavica, 55, 299-308.

Lancashire, S., Haddock, G., Tarrier, N., et al (1996) The impact of training community psychiatric nurses to use psychosocial interventions with people who have severe mental health problems. Psychiatric Services (in press). 
Lewis, S., Tarrier, N., Haddock, G., et al (1995) Inpatient CognitiveBehaviour Therapy in Early Schizophrenia. Wellcome Trust grant application.

Ley, P. (1992) Improving Communication, Satisfaction and Compliance. London: Chapman \& Hall.

Ludwig, W., Huber, D., Schmidt, S., et al (1990) Assessment of compliance related attitudes in psychiatry. A comparison of two questionnaires based on the health belief model. Social Psychiatry and Psychiatric Epidemiology, 25, 298-303.

Margo, A., Hemsley, D. R. \& Slade, P. D. (1981) The effects of varying auditory input on schizophrenic hallucinations. British Journal of Psychiatry, 139, 122-127.

Meichenbaum, D. \& Cameron, R. (1973) Training schizophrenics to talk to themselves: a means of developing attentional control. Behaviour Therapy, 4, 515-534.

Meuser, K. T. \& Berenbaum, H. (1990) Psychodynamic treatment of schizophrenia. Is there a future? Psychological Medicine, 20, 253-262.

Miller, W. R. \& Rollnick, S. (1991) Motivational Interviewing: Preparing People to Change Addictive Behaviour. New York: Guilford Press.

Sellwood, W., Haddock, G. \& Tarrier, N., et al (1994) Advances in the psychological management of positive symptoms of schizophrenia. International Review of Psychiatry, 6, 201-215.

— \& Tarrier, N. (1994) Demographic factors associated with extreme noncompliance in schizophrenia. Social Psychiatry and Psychiatric Epidemiology, 20, 172-177.

Seltzer, A., Roncari, I. \& Garfinkel, P. (1980) Effect of patient education on medication compliance. Canadian Journal of Psychiatry, 25, 638-645.

Slade, P. D. (1972) The effects of systematic desensitisation on auditory hallucinations. Behaviour, Research and Therapy, 10, 85-91.

Tarrier, N., Barrowclough, C., Vaughn, C., et al (1988) The community management of schizophrenia:A controlled trial of behavioural intervention with families to reduce relapse. British Journal of Psychiatry, 153, 532-542.

-_-, Beckett, R., Harwood, S., et al (1993) A trial of two cognitive-behavioural methods of treating drug resistant residual psychotic symptoms in schizophrenic patients: I. Outcome. British Journal of Psychiatry, 162, 524-532.

-, Barrowclough, C., Porceddu, K., et al (1994) The Salford family intervention project: relapse rates of schizophrenia at five and eight years. British Journal of Psychiatry, 165, 829832.

Vaughn, C. E. \& Leff, J. P. (1976) The influence of family life and social factors on the course of psychiatric illness. British Journal of Psychiatry, 129, 125-137.

Wincze, J. P., Leitenberg, H. \& Agras, W. S. (1972) The effects of token reinforcement on the delusional verbal behaviour of chronic paranoid schizophrenics. Journal of Applied Behaviour Analysis, 5, 247-262.

\section{Multiple choice questions}

1. Some of the main outcome measures used in family intervention studies have been:
a Patient symptomatology
b Carer burden
c Finance
d Patient relapse
e Physical health

2. Medication compliance in patients who have a diagnosis of schizophrenia:

a Is less than in patients with long-term

physical illness

b Is greater for medication for physical illness than medication for mental illness

c Has been little studied with regard to medication compliance

d Is more reliably measurable for depot rather than oral medication

e Can be improved with psychological techniques

3. The concept of the 'relapse signature':

a Refers to the changes in writing style observed prior to an acute relapse

b Refers to the symptoms experienced during a relapse

c Refers to the characteristic individual changes which precede an acute relapse

d Predicts the way the patient's symptoms respond to medication

e Requires frequent monitoring to be useful

4. Cognitive-behavioural interventions:

a Have been shown to significantly reduce the severity of persistent positive symptoms

b Should not be used to treat auditory hallucinations

c Have been shown to reliably improve negative symptoms

d Are only effective when used without concurrent medication

e Are ineffective with persecutory delusions

5. Neuropsychological deficits in schizophrenia:

a Have only been shown to occur in a minority of patients

b Are restricted to the frontal lobe

c Are sometimes modifiable using cognitive treatment approaches

d Are wholly remedied by neuroleptic medication

e Are only identifiable following an acute relapse

MCQ answers

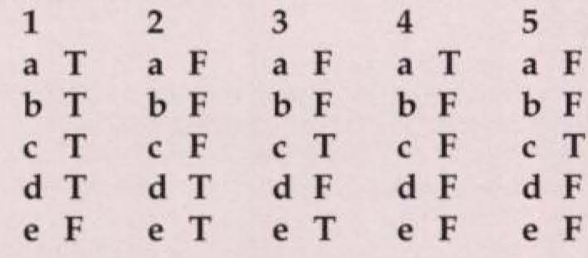

